\title{
Was ist auf dem Weg in die klinische Routine?
}

Fragestellung: Wie weit sind verschiedene Bildgebungsmodalitäten für die Alzheimer-Demenz praxisreif?

Hintergrund: Zur Risikoabschätzung bei Menschen mit einer leichten kognitiven Beeinträchtigung (Mild Cognitive Impairment, MCI, oder DSM-V minor cognitive disorder) oder zur Diagnosestellung bei einer beginnenden Demenz werden zuverlässige Aussagen aus bildgebenden Untersuchungen benötigt.

Patienten und Methodik: Die Autoren suchten systematisch klinische Studien zur Fragestellung und stellten in einer Übersicht diese Ergebnisse sowie ihre Erfahrung aus verschiedenen Forschungsverbünden vor.

Ergebnisse: Die Möglichkeiten des Amyloid-PETs werden bezüglich des spezifischen Nachweises einer auch histologisch später zu sichernden Hirnamyloidablagerung als wissenschaftlich weitgehend valide gesehen. Der Wert dieser Untersuchung für die klinischen Fragen einer Progressionsabschätzung bei MCI oder einer Diagnosesicherung werden allerdings zurückhaltender beurteilt. So ist der Zeitraum einer mit PET nachgewiesenen Hirnamyloidose bis zum Auftreten einer Demenz recht unbestimmt und mit einer Spanne von bis zu 20 Jahren für viele Menschen eventuell nicht entscheidungsrelevant. Für das Glucose-PET und das MRT werden hingegen für die Prognoseabschätzung bei MCI oder die Diagnosestellung bei begin-

Teipel S, Drzezga A, Grothe MJ et al. Multimodal imaging in Alzheimer's disease: validity and usefulness for early detection. Lancet Neurol 2015; 14: 1037 - 53 nender Demenz bereits jetzt gute Voraussetzungen gesehen. Insbesondere die Standardisierung bei flächendeckendem Einsatz und die Trennschärfe bei unselek-

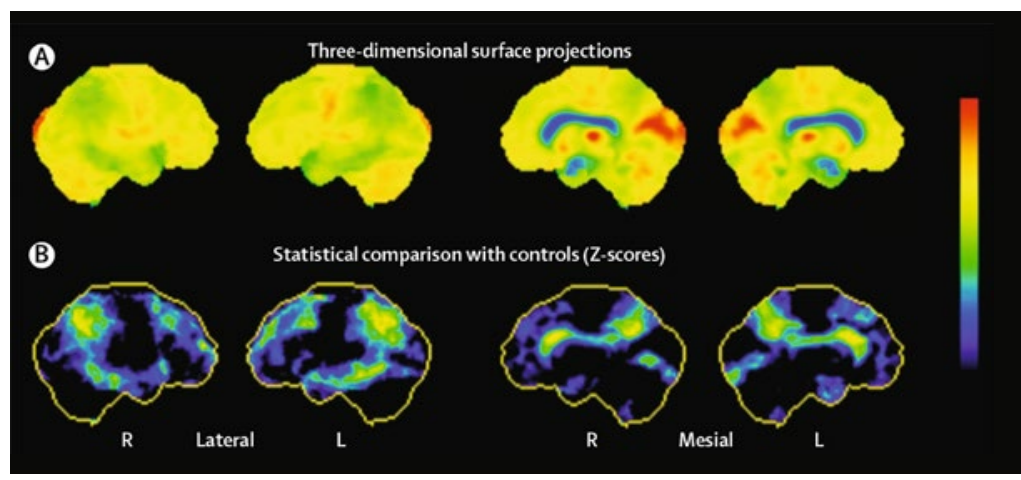

1 Typische Rekonstruktion von Glukose-PET-Daten, die bereits heute klinisch sehr gut interpretiert werden können.

tierten Patienten sowie die Kosten/Nutzen-Frage sind jedoch noch nicht abschließend geklärt.

Die wissenschaftliche Entwicklung mit kombinierten MRT/ PET-Scannern sowie Untersuchungsformen wie DiffusionTensor-Imaging und Resting-State MRI werden als interessante Entwicklungen aufgeführt ( $\triangleright$ Abb. 1).

Schlussfolgerungen: Die Autoren schließen, dass die Progressionsprognose und sichere Differenzialdiagnose insbesondere dann klinisch relevant wird, sobald spezifische krankheitsmodifizierende Therapien für die Alzheimer-Krankheit verfügbar werden. Dann werden auch Fragen der flächendeckenden Implementation und der Kosteneffektivität neu zu beurteilen sein.

\section{- Kommentar von Michael Hüll, Emmendingen}

\section{Diagnostische Möglichkeiten den Therapien weit voraus}

Aus der Übersichtsarbeit wird die Faszination neuer technischer Möglichkeiten spürbar, die insbesondere durch die dynamische Entwicklung der PET-Tracer (tau-Tracer werden auch bald kommen) und der raffinierten mathematischen MRT-Analysen erwachsen. Pragmatisch besteht bereits jetzt in Deutschland eine gute Zugriffsmöglichkeit auf Glukose-PET und MRTAuswertungen, die bei der Frage drohende Progression oder Differenzialdiagnose Alzheimer-Erkrankung nach der Übersichtsarbeit recht gute Dienste leisten. Es fehlen allein noch die therapeutischen Möglichkeiten. Bezüglich der Neuentwicklung von Medikamenten ist in den letzten Jahren doch eine gewisse Ernüchterung eingetreten, was zu einem gewissen Dilemma führt [1]. In den neuen laufenden klinischen Behandlungsstudien werden die ausgefeilten Bildgebungsmodalitäten aber zu einer besseren Wirkstofffindung beitragen.

\author{
Referenzen: \\ 1. Eschweiler GW et al. Dtsch Arztebl Int 2010; 107: 677-83
}

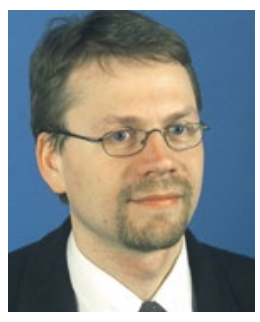

Prof. Dr. med. Michael Hüll, Emmendingen

Klinik für Geronto- und Neuropsychiatrie, Emmendingen

E-Mail:m.huell@zfp-emmendingen.de 